\title{
Pitch as a phonemic cue
}

\author{
PETER HOWELL \\ University College, London WCIE 6BT, England
}

\begin{abstract}
Three experiments that were designed to determine how pitch information is represented in auditory memory are reported. A same-different reaction time was used in all three experiments. Previous experiments have interpreted the finding of faster "same" responses to acoustically identical pairs than to pairs that are phonemically identical but acoustically distinct as indicating that there is a memory that preserves auditory information. It has been assumed that this can be used to match "same" pairs only if the formant frequencies of the members of the pair are the same. In the first experiment, the size of this matching advantage for pairs with identical formant frequencies was not altered when the members of the pair were on different pitches. This indicates that pitch is represented separately from the formants at the auditory level. The second and third experiments used a bigger pitch difference when the pairs were on a different pitch, which, for one of the stimulus sets, resulted in a change in vowel quality but not in the identity of the consonant. In the other stimulus set, both phonemes of the syllable remained the same when presented on different pitches. The matching advantage was reduced when the stimuli were on different pitches for both stimulus sets. This indicates that a difference in pitch can prevent matching at the auditory level under some circumstances. An additional finding, a reduced residual matching advantage when the syllable changes, indicates that at least a syllable-length representation is held in auditory memory. The results are discussed with respect to how the representation in auditory memory might be used in the perception of speech produced by different speakers.
\end{abstract}

During speech perception, the acoustic signal is assumed to be transformed into several different representations during its processing. The representations usually identified are auditory, phonemic, phonological, and semantic. In general, the transformations condense many outputs from previous levels to fewer at the current level. The transformations between levels enable the representations to influence and be influenced by information at later levels (Studdert-Kennedy, 1972). The present paper examines how fundamental pitch is represented at the auditory level and explores how its representation and that of other information in the syllable might assist in the perception of phonemes from different speakers. The pitch of the voice $\left(F_{0}\right)$ is determined by the rate with which the vocal cords vibrate, and it is one means by which the resonances of the vocal tract (the formants) are excited.

One hypothesis about how pitch is represented at the auditory levei was formulated by Morais and Darwin (1974) on the basis of results they obtained from a monaural hemispheric difference experiment. Their experiment and subsequent experiments that have confirmed their findings used a same-different reaction time (RT) task (Darwin, Howell, \& Brady, 1978; Morais, 1975). In these experiments, the subject was required to indicate if a pair of syllables had

\footnotetext{
This work constitutes part of a PhD thesis, and the author was supported by a grant from the S.R.C. I would like to express my thanks to my supervisor, R. J. Audley.
}

the same or a different initial consonant. The standard (first) stimulus was always presented binaurally, and the comparison stimulus on a given trial was presented randomly either to the left or to the right ear. Faster responses were found for stimuli presented to the right rather than to the left ear when the pair was different. For the "same" responses, RTs were faster than for the "different" responses, despite the fact that "same" pairs would seem to require checking that all aspects of a stimulus are the same before a "same" response can be made, whereas "different" pairs can be responded to as soon as any difference is found. There is evidence from matching visually presented letter pairs that decisions can be made on the basis of a representation of the visual trace of the first stimulus that persists for a finite time without recourse to more extensive (and therefore more timeconsuming) categorization processes (Posner, Boies, Eichelman, \& Taylor, 1969). Morais and Darwin (1974) proposed that an auditory representation of the first stimulus was used for matching the "same" pairs in their experiment. Of particular interest with respect to the present investigation is the fact that the stimulus pairs in the Darwin et al. (1978), Morais (1975), and Morais and Darwin (1974) experiments were always presented on different pitches whether the formant frequencies were the same or different.

Despite the pitch (and therefore the waveform) difference in these studies, subjects apparently matched pairs with identical formant frequencies as the same on the basis of their auditory representations. Morais 
and Darwin (1974) hypothesized that the formant frequencies are represented separately from the pitch at the auditory level and that subjects can match the formants irrespective of a difference in pitch at this level. This representation, with only one difference, can then be used to match stimulus pairs as the same at the auditory level.

This account offers no indication of what happens when pitch is used by listeners to identify speech from speakers with different-sized vocal tracts. Since the formant frequencies, to some extent, reflect the vocal tract dimensions, their absolute value is not constant across speakers for the production of the same phoneme. Extra information is needed about the physical dimensions of a speaker's vocal tract to determine what phoneme the speaker intended to produce. Pitch differences are one possible source of information about the size of a speaker's vocal tract, since the pitch variation between speakers is, in general, inversely correlated with vocal tract size. This correlation arises because speakers with small vocal tracts tend to have thinner vocal cords that vibrate at a faster rate (a higher pitch).

The suggestion that pitch information may be used to determine the size of a speaker's vocal tract is supported by the perceptual experiments of Miller (1953). He presented steady-state formant patterns (vowels) on different pitches and found that when these were presented for identification, the phoneme boundaries did not remain constant at the different pitches. Thus the same formant patterns may be categorized as different phonemes, depending on the pitch. It might be supposed that this is because the listener infers the size of the speaker's vocal tract from the voice pitch (see also Fant, Carlson, \& Granstrom, 1974; Wendahl, 1959).

On the one hand, it has been argued that pitch is represented at the auditory level such that it can be discarded for judgments about the sameness or difference of pairs of stimuli. On the other hand, pitch appears to be used as a cue to the size of a speaker's vocal tract, so that the perceiver can determine what phoneme was intended when those formants issue from that vocal tract. In the latter case, pitch should not be discarded at the auditory level. The experiments to be reported examine two questions raised by these studies. First, are Morais and Darwin (1974) correct in proposing that pitch differences do not prevent decisions about formant identity being made on the basis of auditory information? Second, if they are correct, is this true over the range of pitches and formant patterns reported by Miller (1953) to give phoneme boundary shifts? Answers to these questions are interesting because of their implications for the perception of speech from different speakers.

It is necessary to determine if Morais and Darwin's (1974) conclusion that subjects respond "same" at the auditory level irrespective of a difference in pitch is correct. Their conclusion was based on the circum- stantial interpretation of their monaural hemispheric difference experiment. RTs to the "same" pairs in this experiment were faster than those to the "different" pairs, and this was attributed by Morais and Darwin (1974) to matching at the auditory level. However, the difference could be a consequence of "same" responses' not requiring the same amount of execution time as the "different" responses rather than of a literal representation of the first stimulus being used to match identically the "same" pairs of formant frequencies (Egeth, 1966).

The first experiment was designed to test Morais and Darwin's (1974) hypothesis directly. A samedifferent paradigm was employed, using $\mathrm{RT}$ as the dependent variable. Subjects were given a pair of syllables presented in sequence, and they were required to indicate as fast and as accurately as possible whether the consonant of the comparison stimulus was the same as or different from that of the standard stimulus. It has been reported that pairs of stimuli that are acoustically identical are responded to faster than pairs that differ slightly in voice onset time (Pisoni \& Tash, 1974) or in place of articulation (Howell \& Darwin, 1977) but are nevertheless heard as the same phoneme. The difference between these responses (the "same" matching advantage) is reasoned to reflect the difference between the time for matching at the auditory level (identical pairs) and that for matching pairs at the phonemic level (nonidentical "same" pairs). The first experiment was intended to determine whether a difference in pitch between the standard and comparison of approximately the range used by Morais and Darwin (1974) would still permit information at the auditory level to be used for matching when the stimuli were on different pitches. In terms of the analysis of variance, there should be no interaction between "same" matching condition and whether the stimuli are on the same or different pitches. If being on different pitches precludes the subject's ability to match at the auditory level, there should be less advantage when the two items are on different pitches, and an interaction between these factors is expected.

Pisoni and Tash (1974) considered that when the stimuli of the pair are very different, decisions might also be made at the auditory level. This argument has been criticized by Howell and Darwin (1977), Eimas and Miller (Note 1), and Repp (Note 2). For this reason, conclusions about whether variations in pitch between the standard and comparison prevent matching on the basis of auditory identity are only based on "same" responses.

\section{EXPERIMENT 1}

\footnotetext{
Method

Subjects. Eight undergraduate subjects (six female) attended for three sessions, each of which lasted about $1 \mathrm{~h}$. The subjects were between 19 and 25 years of age, and all were right-handed and reported no history of hearing defects. They were paid 60 pence/
} 
session for their participation. The first session was included as practice and is not included in the data analysis. The subject's task in this session was identical in all respects to his final session.

Stimuli. The stimuli were computed by a software parallel formant synthesizer running on a PDP-12 computer. These were output through digital-to-analog converters operating at $8 \mathrm{kHz}$ and filtered at $4 \mathrm{kHz}$ before recording on a Revox tape recorder. All stimuli in this experiment were presented binaurally over headphones at an amplitude of approximately $75 \mathrm{~dB} \mathrm{SPL}$.

Each of the stimuli lasted $200 \mathrm{msec}$; the initial consonant was cued by 40 -msec linear formant transitions. The first formant of all stimuli started at $150 \mathrm{~Hz}$ and rose to a steady-state value of $743 \mathrm{~Hz}$. The starting frequency of the second formant of the first stimulus was $1,232 \mathrm{~Hz}$, and another seven stimuli (eight in all) were synthesized whose starting frequencies increased in steps of $77 \mathrm{~Hz}$. The second formant had a steady state of $1,620 \mathrm{~Hz}$. The third-formant starting frequency of Stimulus 1 was $2,181 \mathrm{~Hz}$, and those of Stimuli $2-7$ increased in $169-\mathrm{Hz}$ steps to $3,195 \mathrm{~Hz}$. The third formant of Stimulus 8 started at $3,026 \mathrm{~Hz}$, and all stimuli had a third-formant steady state of $2,862 \mathrm{~Hz}$. The stimuli were synthesized at constant fundamental frequencies of 120 and $160 \mathrm{~Hz}$, making 16 stimuli in all.

Sequences. Identification sequences of the eight stimuli were prepared. These consisted of a 1,000- $\mathrm{Hz}$ warning tone for $240 \mathrm{msec}$, followed by a 500 -msec pause before one of the stimuli was presented. Each of the eight stimuli occurred five times in random order at each of the two pitches. There was a pause of $3 \mathrm{sec}$ before the next warning tone was presented. Two of these randomizations were prepared.

Stimuli $1,3,6$, and 8 were used in the same-different matching task. In other experiments, these were found to be readily identified as either /bae/ (Stimuli 1 and 3) or /dae/ (Stimuli 6 and 8). The pairs of stimuli for the same-different task were prepared by pairing each of the stimuli as the standard with each of the stimuli as comparison three times. These formant patterns were presented at each of the four pitch combinations of standard and comparison (high-high, high-low, low-high, low-low), making 192 trials in all. The stimulus pairs were randomized, and the sequences were recorded with a warning tone followed by a $500-\mathrm{msec}$ pause before the standard and a 200 -msec pause before the comparison. Stimulus onset asynchrony (SOA) in this experiment was $400 \mathrm{msec}$. Between each of these pairs there was a pause of $3 \mathrm{sec}$. Three different randomized blocks were recorded.

Experimental design. At the beginning of each session, subjects received one of the randomizations of the 80 -item identification recordings. Subjects indicated whether they heard the stimuli as /bae/ or /dae/, irrespective of the pitch of the stimulus, by pressing one of two response keys with the index fingers of each hand. Subjects indicated this categorization with the same key assignment throughout, but this was counterbalanced across subjects. The response category chosen was stored for analysis purposes.

In the same-different matching task, the subject was required to indicate if the second stimulus was the same as or different from the first, irrespective of the pitch. The subject was required to indicate consonant category identity, not identity of the formant transitions. The subject was instructed to do this as quickly and as accurately as possible, using a lever operated by one hand. Half the subjects pulled the lever toward or pushed it away from them to indicate "same" or "different," and the other half operated the key in the reverse directions for corresponding responses. Each subject received all three randomizations (referred to as "blocks") during each session, and the order with which the subject received these randomizations was counterbalanced across sessions and across subjects. The hand the subject used was counterbalanced between blocks. The response the subject made and the time for this decision measured from the onset of the comparison stimulus were recorded and stored for later analysis by a PDP-12 computer.
Results

The subjects' categorization of the stimuli in the identification task are shown in Figure 1. Subjects assigned the stimuli used in the classification task to the expected response categories better than $96 \%$ of the time, Stimuli 1 and 3 to /bae/ and Stimuli 6 and 8 to $/$ dae/.

The mean correct RT of Sessions 2 and 3 and the number of errors for the experimental conditions were computed for each block of each session. The stimulus pairing gives rise to "different" pairs that differ to varying extents with respect to their secondformant starting frequencies. The "different" stimulus pairs were partitioned into three-, five-, and sevenstep differences, where "step" refers to the number of stimuli that the stimuli of the pair were separated by on the original (eight-token) stimulus continuum. In the analyses performed, mean correct RTs were not transformed, but error rates were subjected to an arcsin root transformation. Analyses of variance were performed with the factors (1) subjects, (2) whether the pitches of the first and second stimuli were the same or different, (3) whether the pitch of the second stimulus was high or low, (4) sessions, (5) within-sessions replications (i.e., the three randomized blocks that the subject received within a session), and (6) matching conditions (identical vs. nonidentical "same" for "same" responses and steps of difference for the "different" responses). The mean RTs of the experimental conditions of interest are shown in Figure 2.

Inspection of the RTs of the "same" responses presented in Figure 2 shows that the RT to identical pairs was faster than the nonidentical "same" responses. This impression was confirmed by statistical analysis. Thus the main effect of matching conditions was significant $[F(1,7)=8.9, p<.05]$. As outlined in the introduction, this is reasoned to reflect the difference between making decisions at the auditory and phonemic levels. Furthermore, the advantage

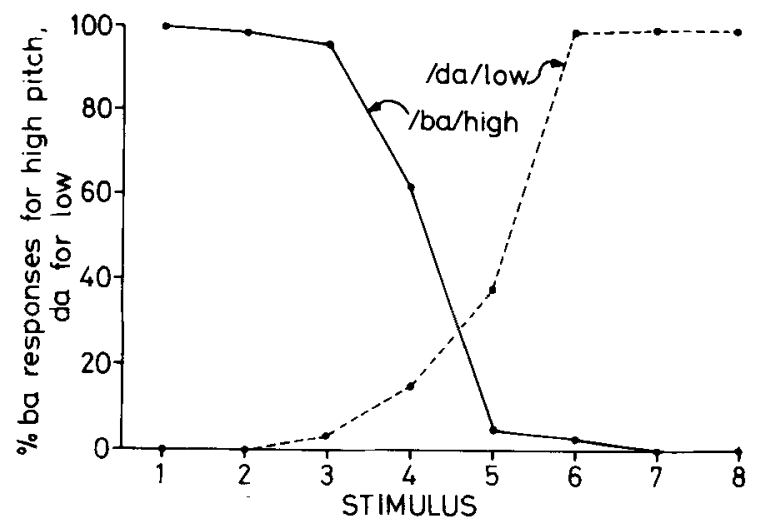

Figure 1. Percentage identification of the eight stimuli as /ba/ for low pitch, /da/for high pitch for the subjects in Experiment 1. 
appears to be as large whether the members of the pair are on the same or different pitches. As predicted by Morais and Darwin's (1974) hypothesis, auditory matches can apparently be made as fast when the members of the pair are on different pitches as when they are on the same pitch. The lack of an interaction in the analysis of variance between pitch and matching condition supported this conclusion.

As reported by Howell and Darwin (1977) and Pisoni and Tash (1974), the "different" responses showed a tendency for faster responding when there was a large acoustic difference between the members of the pair $[F(2,14)=11.9, p<.001]$. Since Pisoni and Tash (1974) attributed this effect to large acoustic differences enabling matching at the auditory level, the results of the analysis of variance were examined to see whether this tendency differed when the members of the pair were on the same or different pitches. As with the RTs of the "same" responses, there was no pitch effect, nor were any of the interactions with this factor significant.

It is possible to achieve a RT difference between conditions by selectively relaxing the accuracy cri-
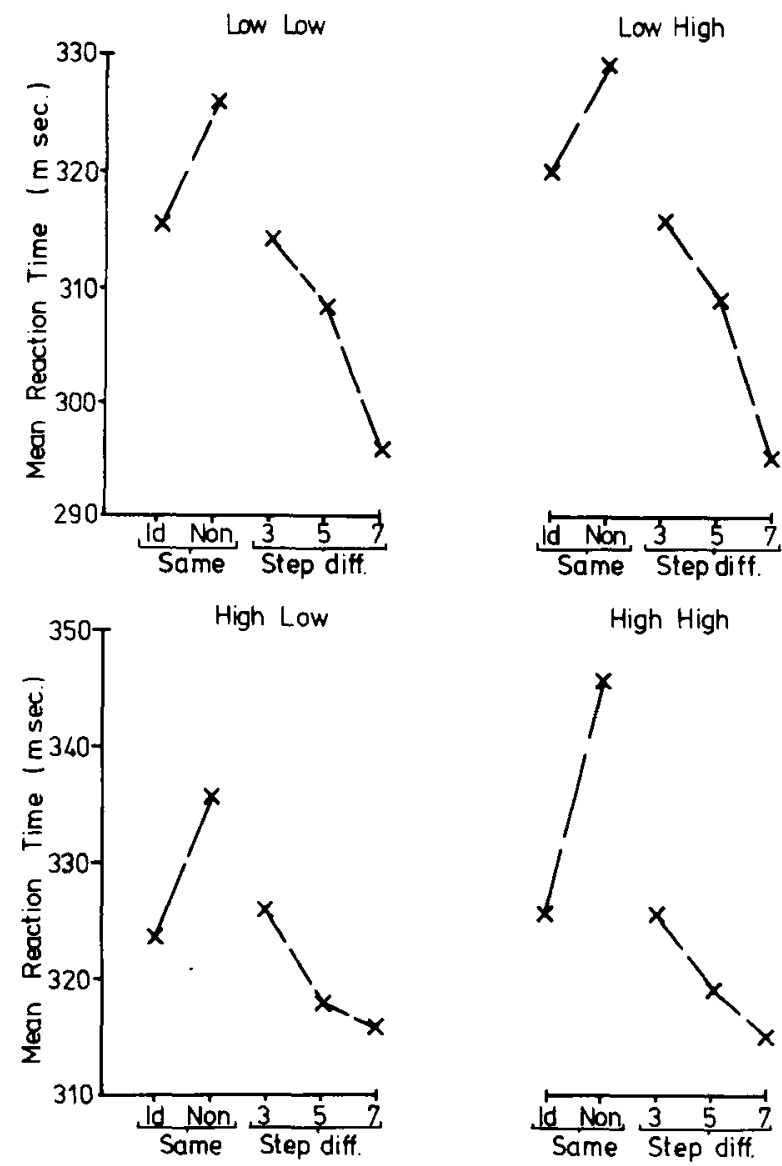

Figure 2. RTs for the matching conditions in Experiment 1. The label of each quadrant refers to the pitch of the first and second stimulus, respectively.
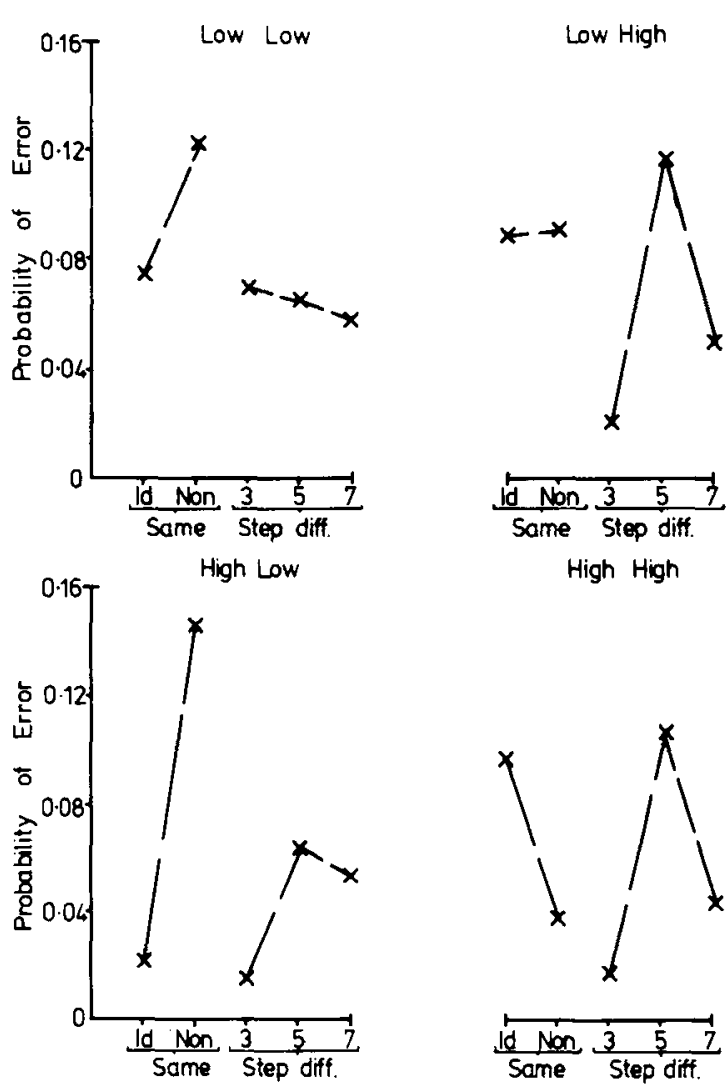

Figure 3. Error proportions for the matching conditions in Experiment 1. The label of each quadrant refers to the pitch of the first and second stimulus, respectively.

terion. However, when the error rates (which are presented in Figure 3) were analyzed, there were no statistically significant differences across matching conditions for "same" or "different" responses. This indicates that subjects were not trading speed for accuracy.

\section{Discussion}

The main effect of the "same" matching condition indicates that subjects are able to match identical pairs faster than acoustically nonidentical pairs from the same phonemic category. The advantage for identical pairs may be taken as evidence for an auditory memory. The lack of an interaction with whether the stimuli are on the same or different pitches shows that the matching advantage is as large when stimulus pairs differ in pitch as when they do not. The results of the present experiment indicate that the pitch of the speech sound in auditory memory apparently does not preclude auditory information's being used to determine whether stimulus pairs are the same when they are on different pitches, despite the fact that the pitch difference is heard clearly by all subjects. This confirms the prediction derived from Morais and Darwin's (1974) interpretation of their monaural hemispheric difference experiment. Their 
results were interpreted as indicating that pitch information is represented separately from the formants at the auditory level.

As outlined in the introduction, Miller (1953) reported that presenting the formant frequencies of vowel stimuli on different pitches may result in a change in phoneme identification. If subjects can match formant frequencies to be the same at the auditory level when there is a small pitch difference, how is this ability affected by a pitch difference as large as that used by Miller (1953)? In particular, how can such information be used when the pitch variation gives rise to a change in the phoneme? If the latter speech sounds are matched at the auditory level, irrespective of a difference in pitch, then information that contributes to the identity of the phoneme must be discarded.

Experiments 2 and 3 replicated Experiment 1, but they employed a larger pitch difference than that in Experiment 1 for stimuli presented on different pitches. The subject's task was to match the consonants, and two stimulus sets were used. In the first stimulus set, the vowel changed when stimuli that might otherwise have been the same were presented on different pitches (varying vowel set). In the second set, an equivalent pitch difference was employed, but the vowel did not change (constant vowel set). Vowel changes were used in the stimulus sets for two reasons: First, pilot experiments indicated that these changes are more easily achieved than consonant changes, and second, Howell (1978) concluded that the auditory representation is matched on syllables, rather than on the phoneme segments selected by the matching instructions. In Howell's experiment, stop consonants had to be matched when they were cued by the same formant transitions before different vowels. By varying the irrelevant segment in the to-be-matched syllable, this finding can be extended. In particular, the role of the irrelevant segment in determining the articulatory dimensions of a speaker's vocal tract can be examined.

It was expected that if the larger range of pitch differences tends to prevent matching on the auditory representation, then the matching advantage would be reduced for both stimulus sets when they were presented on different pitches. If, in addition to the pitch range's tending to prevent auditory matching, a syllable is the unit that is represented in auditory memory, then the change in vowel quality caused by a difference in pitch should result in less matching advantage for the varying vowel stimuli when they are on different pitches than for the constant vowel set.

An additional design change was made because the "same" matching advantage was not very large in Experiment $1(15 \mathrm{msec})$ : It has been shown that this advantage disappears as the SOA increases
(Howell, 1978; Howell \& Darwin, 1977; Eimas \& Miller, Note 1). In order to increase the matching advantage, the duration of the vowel was $100 \mathrm{msec}$ less, giving an SOA of $200 \mathrm{msec}$.

\section{EXPERIMENT 2}

\section{Method}

Subjects. Sixteen subjects (six male) from the same source as Experiment 1 and between 18 and 23 years of age attended for three sessions of about $1 \mathrm{~h}$ each. They received a prorata payment of 60 pence/session. None reported any history of hearing defects, and all said that they were right-handed. Since the experiment required identifying synthetic speech for vowel quality and consonant at different pitches, subjects were used who had previous experience with such speech. Eight of the subjects performed on the varying vowel set, and the remainder, on the constant vowel set.

Stimuli. Two sets of stimuli were synthesized: One set was expected to give different vowels when presented at pitches of 140 and $280 \mathrm{~Hz}$ (varying vowel set); the other set was not expected to give a change in the vowel (constant vowel set). Both sets consisted of two-formant patterns, and each set of formant patterns was synthesized at pitches of 140 and $280 \mathrm{~Hz}$, with formant transitions approaching the vowel steady state linearly over $40 \mathrm{msec}$. The vowel portion lasted for $60 \mathrm{msec}$.

For the varying vowel set, the first formant of each stimulus started at $150 \mathrm{~Hz}$ and rose to a steady state of $850 \mathrm{~Hz}$. For the second formant, the steady state was always $1,250 \mathrm{~Hz}$. Four consonants, varying in the second-formant starting frequency, were synthesized. Two stimuli were heard as / $b /$ (starting frequencies of $970 \mathrm{~Hz}$ for $\mathrm{BL}$ and $1,005 \mathrm{~Hz}$ for $\mathrm{BH}$ ). Two additional stimuli with starting values of $1,530 \mathrm{~Hz}(\mathrm{DH})$ and $1,495 \mathrm{~Hz}(\mathrm{DL})$ were heard as $/ \mathrm{d} /$.

For the constant vowel set, the first formant always started at $150 \mathrm{~Hz}$ and had a steady state of $850 \mathrm{~Hz}$. The second-formant starting frequencies were the same as for the varying vowel set, and their designation was the same as the varying vowel set. The steady state of the second formant was $1,600 \mathrm{~Hz}$. The consonant syntheses in the vowel sets corresponded approximately to a common locus frequency for stop consonants before different vowels (Liberman, Cooper, Shankweiler, \& Studdert-Kennedy, 1967).

Sequences. Each identification sequence consisted of 10 presentations of each stimulus at each pitch in random order and separated by $3 \mathrm{sec}$. The same-different sequences consisted of 192 trials randomized as in Experiment 1. The same-different recordings were made with a 100 -msec pause between the standard and the comparison and $3 \mathrm{sec}$ between pairs ( $\mathrm{SOA}=200 \mathrm{msec}$ ).

Experimental design. Before the same-different task, the subject received the identification task with the appropriate stimulus set. These identification tasks differed with respect to the syllable categories to which the subject was allowed to assign the stimuli. For the varying vowel set, subjects were required to classify the stimuli as $/ \mathrm{ba} /, / \mathrm{da} /, / \mathrm{b} N /$, or $/ \mathrm{d} \Lambda /$. For the constant vowel set, subjects were required to classify the stimuli as $/ \mathrm{bae} /, / \mathrm{b} N$, $/ \mathrm{b} \varepsilon /, / \mathrm{dae} /, / \mathrm{d} \Lambda /$, or $/ \mathrm{d} \varepsilon /$ after they had been given illustrations of words in which these phonemes occurred. These stimulus categories were chosen either because they were the reported vowel change (varying vowel set) or because they were adjacent to the formant patterns in Miller's (1953) confusion matrix (constant vowel set).

\section{Results}

The results were analyzed in the same way as in Experiment 1. The results of the identification tasks are shown in Tables 1 and 2 for the varying and con- 
Table 1

Percent Stimulus Categorization of the Varying Vowel Set Stimuli Presented for Identification in Experiment 2

\begin{tabular}{crrrr}
\hline & \multicolumn{4}{c}{ Stimulus } \\
\cline { 2 - 5 } Response & BL & \multicolumn{1}{c}{ BH } & \multicolumn{1}{c}{ DL } & \multicolumn{1}{c}{ DH } \\
\hline BA & 98.2 & 3.2 & .8 & .3 \\
DA & .2 & .8 & 96.5 & 6.2 \\
BU & 1.3 & 95.4 & .7 & .3 \\
DU & .3 & .6 & 2.0 & 93.2 \\
\hline
\end{tabular}

Note-The first letter of the stimulus symbols indicates the expected stop consonant; the second symbol indicates whether the stimulus was on a low $(140 \cdot \mathrm{Hz})$ or high $(280 \cdot \mathrm{Hz})$ pitch. Each stimulus was assigned to the response classes on the left of the table (BA, DA, $B U$, or $D U)$.

Table 2

Percent Stimulus Categorization of the Constant Vowel Set Stimuli Presented for Identification in Experiment 2

\begin{tabular}{lrrrr}
\hline & \multicolumn{4}{c}{ Stimulus } \\
\cline { 2 - 5 } Response & \multicolumn{1}{c}{$\mathrm{BL}$} & $\mathrm{BH}$ & \multicolumn{1}{c}{$\mathrm{DL}$} & $\mathrm{DH}$ \\
\hline /bae/ & 94.2 & 96.3 & 3.3 & 2.3 \\
$/ \mathrm{b} \Lambda /$ & 1.2 & .9 & .3 & .0 \\
$/ \mathrm{b} \epsilon /$ & .6 & .0 & .0 & .0 \\
$/ \mathrm{dae} /$ & 3.4 & 2.1 & 95.4 & 97.7 \\
$/ \mathrm{d} \Lambda /$ & .6 & .7 & 1.0 & .0 \\
$/ \mathrm{d} \epsilon /$ & .0 & .0 & .0 & .0 \\
\hline
\end{tabular}

Note-The first letter of the stimulus symbols indicates the expected stop consonant; the second symbol indicates whether the stimulus was on a low $(140-\mathrm{Hz})$ or high $(280-\mathrm{Hz})$ pitch. Each stimulus was assigned to the response classes on the left of the table.

stant vowel sets, respectively. These indicate that subjects classified the stimuli into the expected response classes.

Inspection of the "same" RTs of the varying vowel set, which are presented in Figure 4, shows that there is a "same" matching advantage when the stimuli are on the same pitch but none when they are on different pitches. This was confirmed by the presence of a main effect of matching condition $[F(1,7)=6.1, p<.05]$, which indicates that, overall, there was a matching advantage, and by the interaction with whether the stimulus pairs were on the same or different pitches $[F(1,7)=8.8, p<.05]$, which indicates that there was a larger matching advantage for pairs that were on the same pitch.

A similar pattern of results was observed with the "same" RTs of the constant vowel set, which are presented in Figure 5. Thus there was a main effect of matching condition $[\mathrm{F}(1,7)=10.6, \mathrm{p}<.01]$ and an interaction of whether the stimuli were on the same or different pitches and matching conditions $[F(1,7)=54.2, p<.001]$.

The matching advantage when the stimuli were on the same pitch was approximately the same across the two vowel conditions (30 and $34 \mathrm{msec}$ in the varying and constant vowel sets, respectively), but the advantage was virtually absent when the items were on different pitches. For the varying vowel set, the advantage was only $1 \mathrm{msec}$, but there was still a 10-msec advantage for the constant vowel set. An analysis of variance with the extra factor of vowel set included indicated that the reduction when the pairs were different was greater for the varying than for the constant vowel set $[F(1,7)=8.9, p<.025]$.

In none of the analyses of the "different" RTs nor in any of the analyses of error rates was there any indication that the effect of matching conditions differed when the members of the pair were on the same or on a different pitch. The error rates for the varying vowel set are presented in Figure 6; those for the constant vowel set are presented in Figure 7.

\section{Discussion}

The results for "same" RTs suggest that the ability to use the auditory representation to match pairs with identical formant frequencies is reduced when the

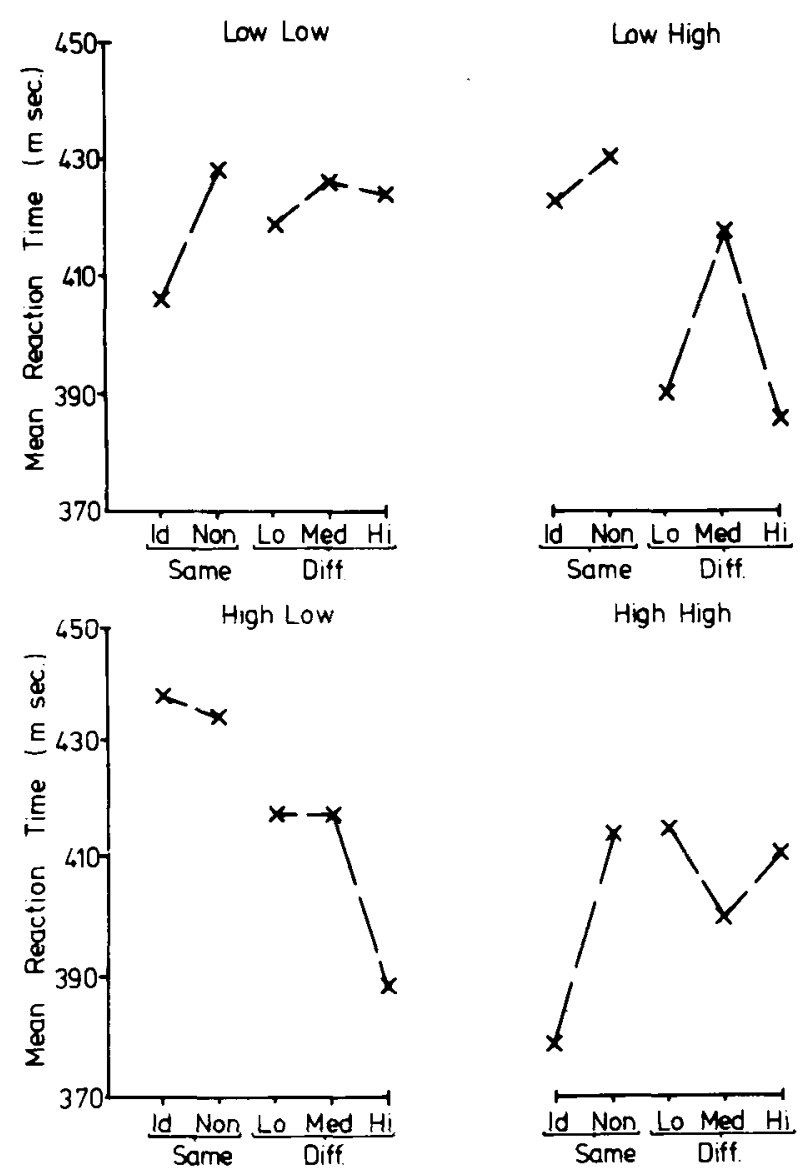

Figure 4. RTs for the matching condition in the varying vowel set of Experiment 2. The label of each quadrant refers to the pitch of the first and second stimulus, respectively. $\mathrm{Hi}$, Med, and Lo refer to the amount of acoustic difference, as outlined in the text. 


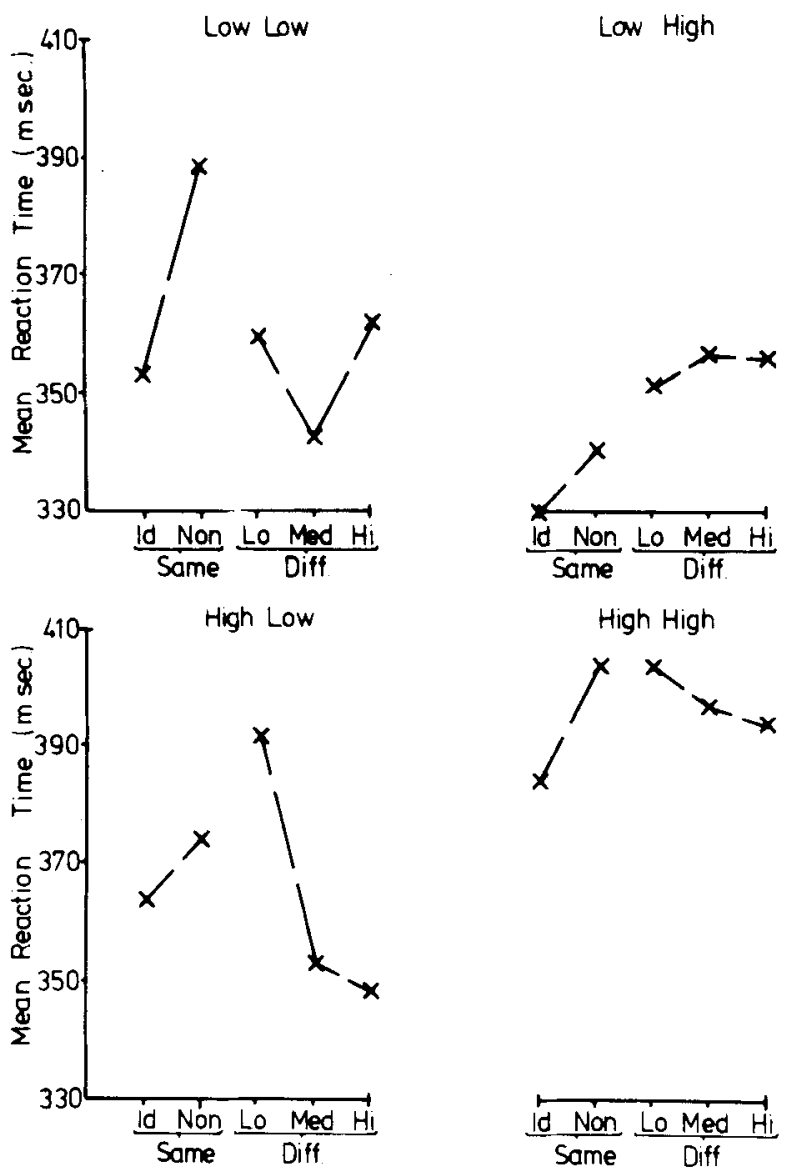

Figure 5. RTs for the matching condition in the constant vowel set of Experiment 2. The label of each quadrant refers to the pitch of the first and second stimulus, respectively. Hi, Med, and Lo refer to the amount of acoustic difference, as outlined in the text.

stimuli are on different pitches. In addition, there is some residual matching advantage for the constant vowel set but none for the varying vowel set. Before the implications of the results are discussed further, the next experiment will be presented. Experiment 3 was performed to confirm the findings of Experiment 2. One extra design change was made: Both the constant and the varying vowel stimulus conditions were performed by each subject.

\section{EXPERIMENT 3}

\section{Method}

Subjects. Eight subjects (five male) from the same source as in the previous experiment and between the ages of 19 and 26 years attended for five sessions of about $1 \mathrm{~h}$ each. They received a prorata payment of 60 pence/session. None reported any history of hearing defects, and all said that they were right-handed. Practiced subjects were used, as in Experiment 2.

Stimuli and sequences. The stimuli and sequences used were the same as those in Experiment 2.

Experimental design. The subject performed the practice session, consisting of both identification tests and one same-different sequence of each of the vowel sets. In the experimental sessions, one group of four subjects was tested with the varying vowel set first and the other with the constant vowel set first. The subjects performed on one vowel set within a session and received both sessions on one vowel set before performing on the other.

\section{Results}

The identification results are presented in Table 3 for the varying vowel set and in Table 4 for the constant vowel set. The results indicate that the stimuli were assigned to the expected response classes again.

The RTs were analyzed in the same way as in Experiments 1 and 2. The RTs for the varying vowel set are shown in Figure 8; those for the constant vowel set are shown in Figure 9.

The results of the "same" RTs present the same picture as that found in Experiment 2. There was a "same" matching advantage for both the constant and varying vowel sets $[F(1,7)=74.3, p<.001$, and $\mathrm{F}(1,7)=24.0, \mathrm{p}<.01$, respectively]. The matching advantage was reduced when the stimuli were on
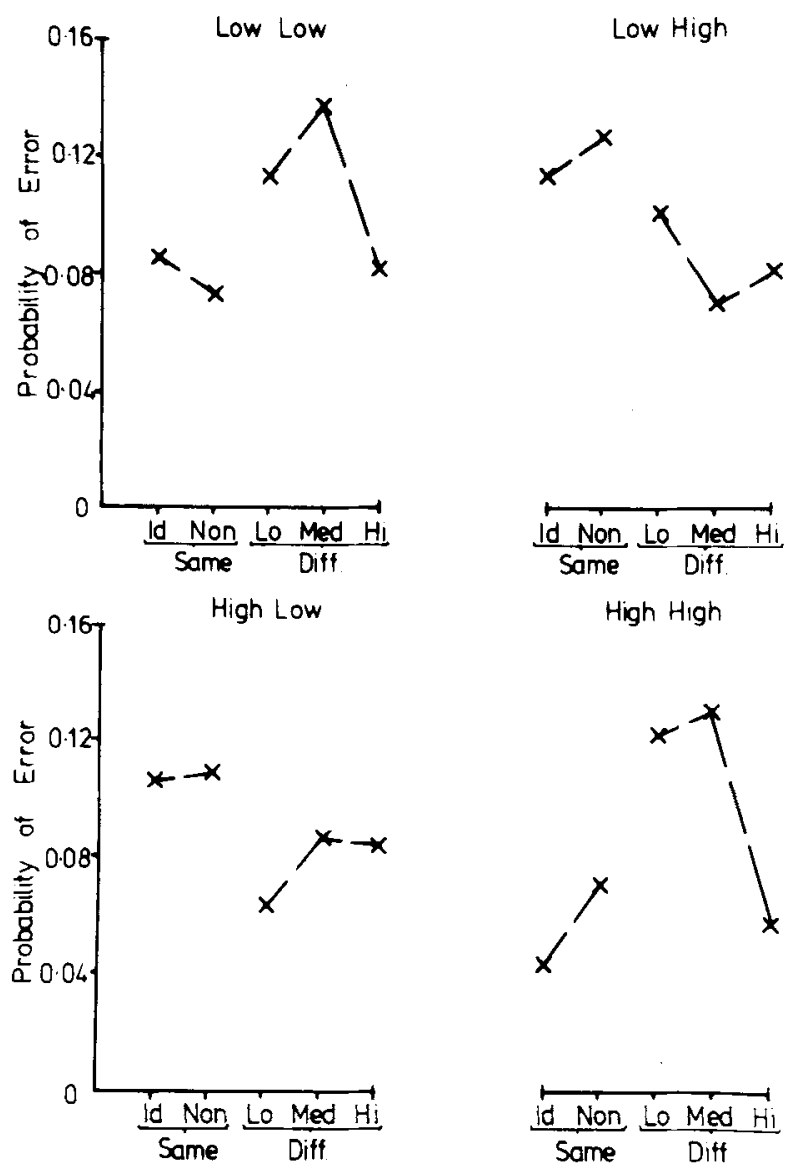

Figure 6. Error proportions for the matching conditions in the varying vowel set of Experiment 2. The label of each quadrant refers to the pitch of the first and second stimulus, respectively. $\mathrm{Hi}$, Med, and Lo refer to the amount of acoustic difference, as outlined in the text. 

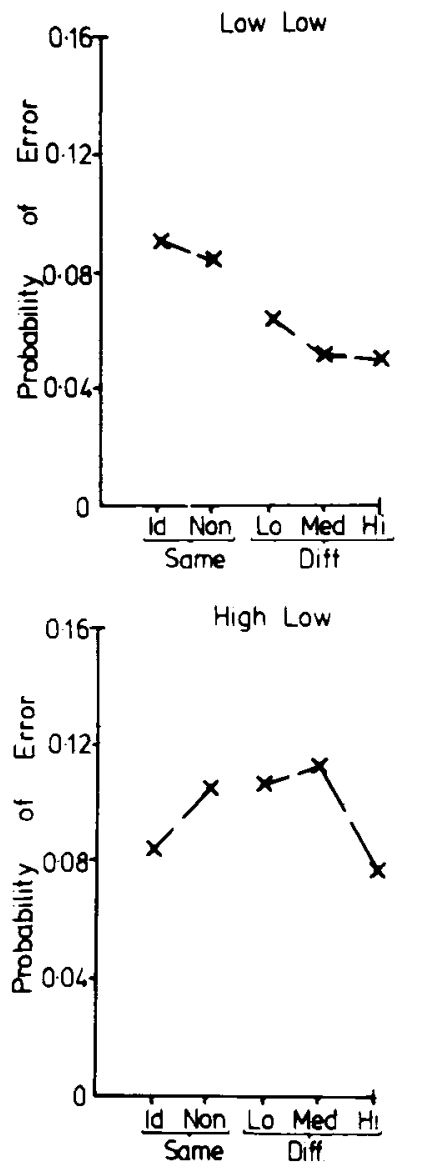

Figure 7. Error proportions for the matching conditions in the constant vowel set of Experiment 2. The label of each quadrant refers to the pitch of the first and second stimulus, respectively. Hi, Med, and Lo refer to the amount of acoustic difference, as outlined in the text.

different pitches: The interaction between matching condition and same or different pitch was significant for both vowel sets $[F(1,7)=69.7, p<.001$, and $F(1,7)=29.6, p<.01$, for the varying and constant vowel sets, respectively]. Analysis of the two vowel sets together showed that the reduction in the "same" matching advantage was greater for the varying vowel set again: The interaction among vowel set, matching condition, and whether the pair members were on the same or on a different pitch was significant $[F(1,7)=6.3, p<.05]$.

Analysis of the error rates, presented in Figures 10 and 11 , for the varying and constant vowel sets, respectively, showed that there was an interaction between matching condition with the same or different pitch for the "same" and "different" responses $[\mathrm{F}(1,7=105.5, \mathrm{p}<.001$, and $\mathrm{F}(2,14)=6.7, \mathrm{p}<.01$, respectively]. For the "same" and "different" pairs, this could have led to an underestimation of the matching advantage when the stimuli were on different pitches, but it is of note that there was no inter- action with vowel condition. The "different" RTs showed no interaction between matching condition and whether the members of the pair were on the same or on different pitches.

\section{Discussion}

The overall matching advantage found for identical pairs as opposed to nonidentical "same" pairs is again taken as evidence for a literal representation of the first stimulus that can be used for matching the identical "same" pairs. In Experiments 2 and 3, and contrary to the results of Experiment 1, the interaction between matching condition and whether the pair members were on the same or different pitches indicates that a difference in pitches between the members of the pair restricts auditory information being used to determine whether two stimuli are the same when they have identical formant frequencies. The presence of the interaction with vowel set shows that the "same" matching advantage is restricted further, depending on whether the vowel of the syllable changes with pitch. From these results it is concluded that the use of a pitch difference larger than that used in Experiment 1 restricted the use of infor-

Table 3

Percent Stimulus Categorization of the Varying Vowel Set Stimuli Presented for Identification in Experiment 3

\begin{tabular}{crrrr}
\hline & \multicolumn{4}{c}{ Stimulus } \\
\cline { 2 - 5 } Response & \multicolumn{1}{c}{ BL } & \multicolumn{1}{c}{ BH } & \multicolumn{1}{c}{ DL } & \multicolumn{1}{c}{ DH } \\
\hline BA & 96.9 & 4.7 & .2 & .6 \\
DA & .2 & .8 & 93.8 & 4.0 \\
BU & 2.5 & 94.3 & .6 & .3 \\
DU & .4 & .2 & 5.4 & 95.1 \\
\hline
\end{tabular}

Note-The first letter of the stimulus symbols indicates the expected stop consonant; the second symbol indicates whether the stimulus was on a low $(140-\mathrm{Hz})$ or high $(280-\mathrm{Hz})$ pitch. Each stimulus was assigned to the response classes on the left of the table ( $B A, D A, B U$, or $D U)$.

Table 4

Percent Stimulus Categorization of the Constant Vowel Set Stimuli Presented for Identification in Experiment 3

\begin{tabular}{lrrrr}
\hline & \multicolumn{4}{c}{ Stimulus } \\
\cline { 2 - 5 } Response & BL & BH & DL & \multicolumn{1}{c}{ DH } \\
\hline /bae/ & 93.0 & 94.2 & 1.2 & 5.2 \\
$/ \mathrm{b} \Lambda /$ & .9 & .8 & 2.4 & .6 \\
$/ \mathrm{b} \epsilon /$ & .7 & .5 & .4 & .3 \\
$/ \mathrm{dae} /$ & 4.9 & 3.8 & 96.0 & 93.7 \\
$/ \mathrm{d} \Lambda /$ & .2 & .4 & .0 & .2 \\
$/ \mathrm{d} \epsilon /$ & .3 & .3 & .0 & .0 \\
\hline
\end{tabular}

Note-The first letter of the stimulus symbols indicates the expected stop consonant; the second symbol indicates whether the stimulus was on a low (140-Hz) or high (280-Hz) pitch. Each stimulus was assigned to the response classes on the left of the table. 

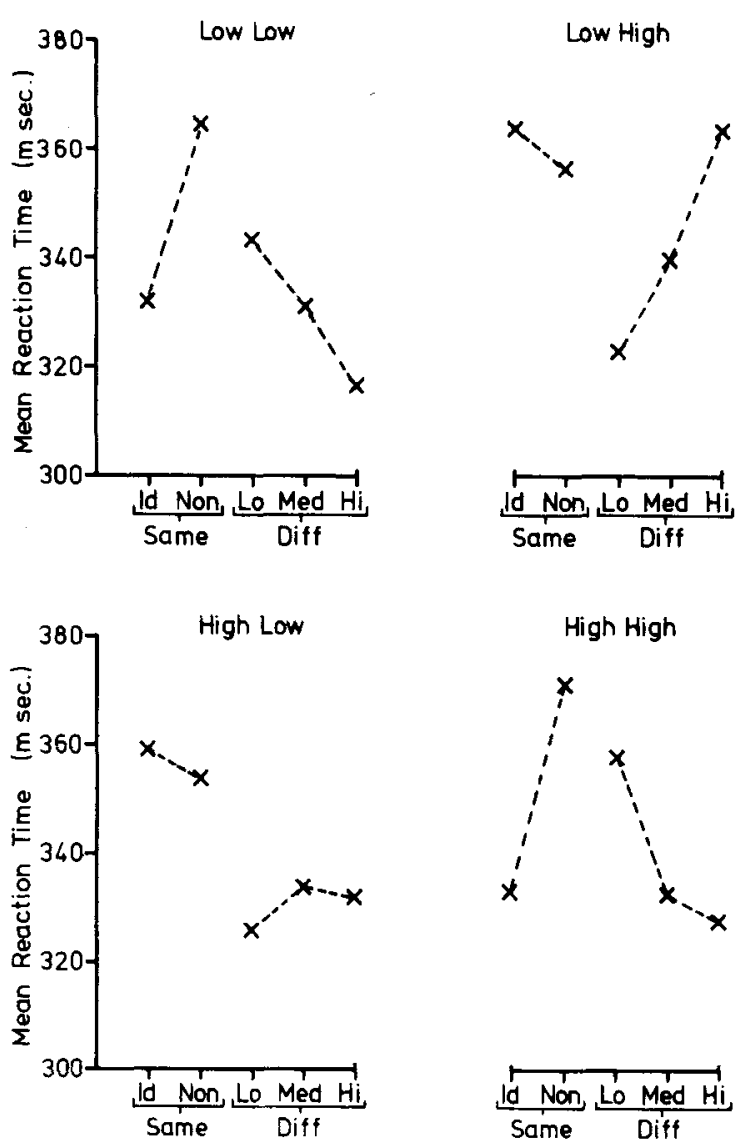

Figure 8. RTs for the matching conditions in the varying vowel set of Experiment 3. The label of each quadrant refers to the pitch of the first and second stimulus, respectively. Hi, Med, and Lo refer to the amount of acoustic difference, as outlined in the text.

mation at the auditory level for matching and that this restriction was greater when the vowels of the syllables differed. Note that the latter finding is true even though consonant matching was required.

\section{GENERAL DISCUSSION}

The experiments reported here reveal two main findings: The first experiment shows that when a pair of stimuli are to be matched, the advantage of identical "same" pairs and phonemically "same" pairs that differ slightly in formant frequency is as large whether the items are on the same or on different pitches. As outlined in the introduction, Morais and Darwin (1974) reasoned that pitch is represented separately from the formants at the auditory level. This would allow pairs that differ in pitch to be matched as the same on the basis of the auditory representation. This account predicts the results of Experiment 1. The second and third experiments demonstrate that pitch variation between the members
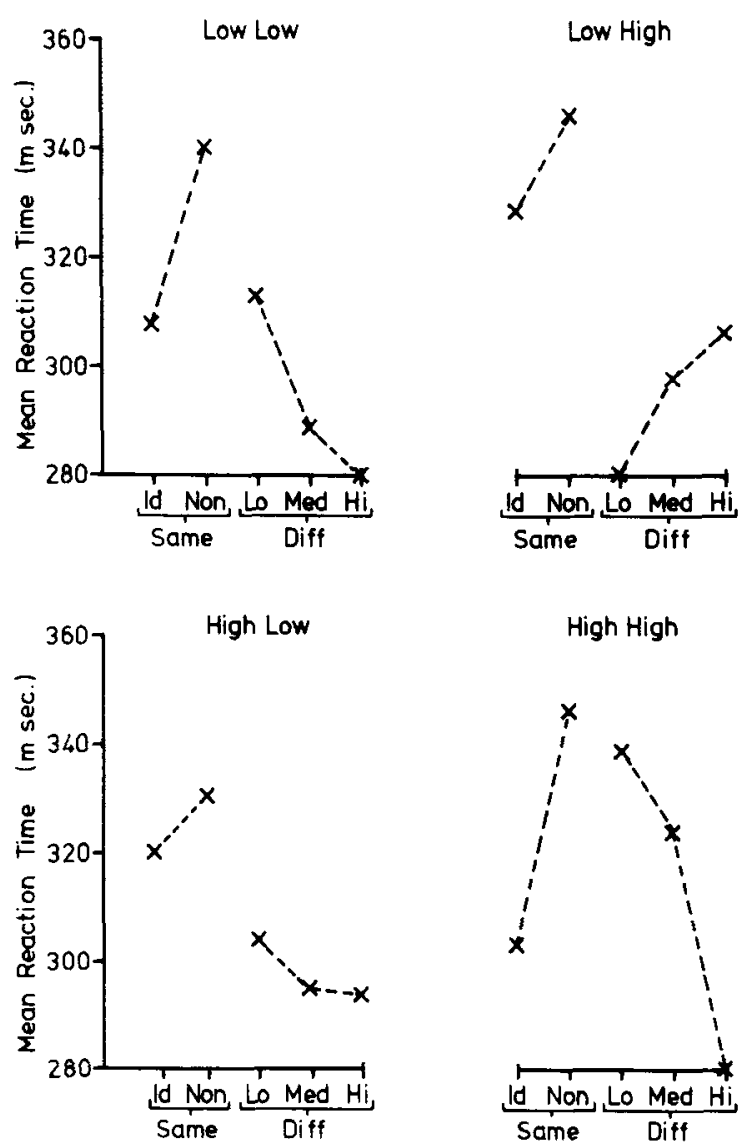

Figure 9. RTs for the matching condition in the constant vowel set of Experiment 3. The label of each quadrant refers to the pitch of the first and second stimulus, respectively. Hi, Med, and Lo refer to the amount of acoustic difference, as outlined in the text.

of a pair may abolish the matching advantage when a larger pitch range is used. It will be shown below how the system could give rise to these effects. The attenuation of the matching advantage in Experiments 2 and 3 is more marked when the vowel of the syllable is identified as a different vowel on the different pitches. Matching depends on the properties of the syllable rather than on the phoneme segment selected by the matching instructions. In this section, the implications of these results for the characteristics of auditory memory that might be useful in enabling phonemic processing to deal efficiently with speech from different speakers is examined.

Three questions are raised: First, if syllable information is available in auditory memory, what advantage would this offer for the processing of phonemes produced by different speakers? Second, what advantage would the representation of pitch separately from the formant frequencies offer for the identification of phonemes from different speakers? The third question is whether changing the percep- 

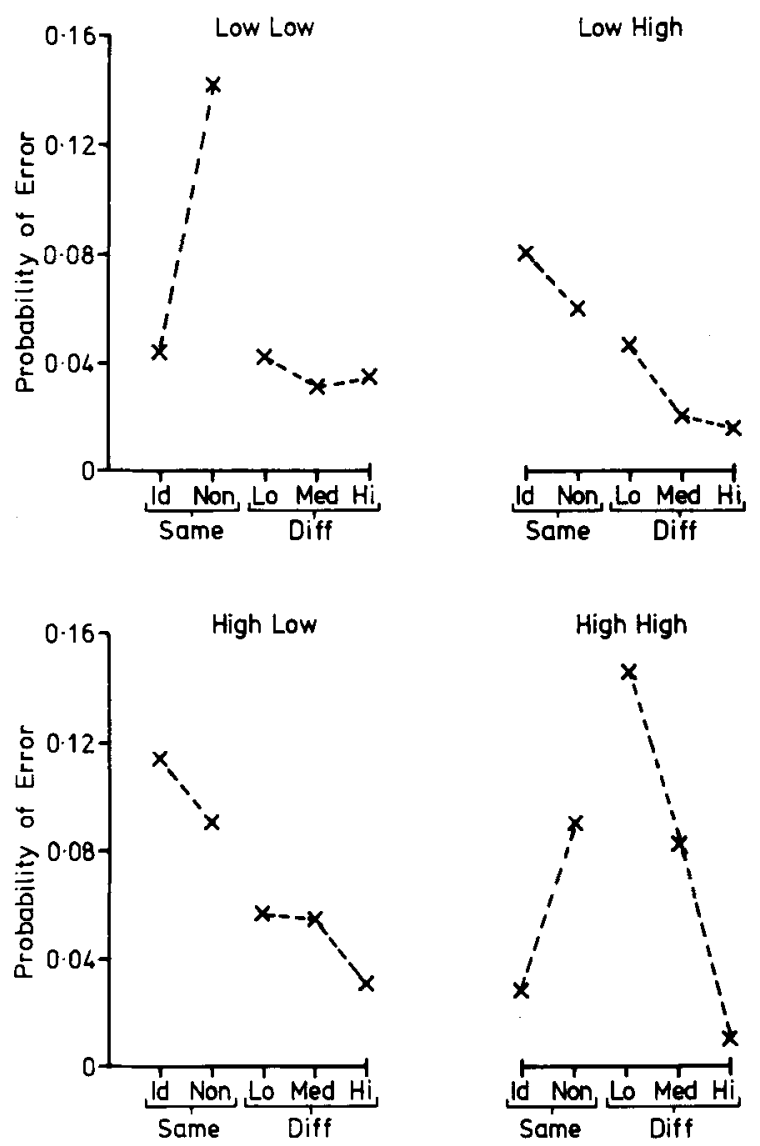

Figure 10. Error proportions for the matching conditions in the varying vowel set of Experiment 3. The label of each quadrant refers to the pitch of the first and second stimulus, respectively. Hi, Med, and Lo refer to the amount of acoustic difference, as outlined in the text.

tual evaluation of the acoustic cues when the speaker changes requires a separate mechanism to recalibrate the phonemic mechanism. The perceptual mechanism could be recalibrated by scaling the formant frequencies for an assumed vocal tract length for a speaker with that voice pitch.

The findings in Experiments 2 and 3 that the "same" matching advantage is attenuated with a large pitch range and is further attenuated when the vowel changes suggests that the syllable is being used for matching at the auditory level. The conclusion that syllabic or greater length information is held in auditory memory was drawn in a recent experiment by Howell (1978). The irrelevant segment in that experiment was varied by employing consonant-diphthong syllables in which the diphthong formants were cued only by the direction and rate of change (not starting value) of the second formant. The consonant transitions to be matched could be the same before different diphthongs. It was found that even when only the segment irrelevant to the matching decision was varied, the "same" matching advantage was abolished. Thus matching depended on the properties of the syllable rather than on the properties of the phoneme.
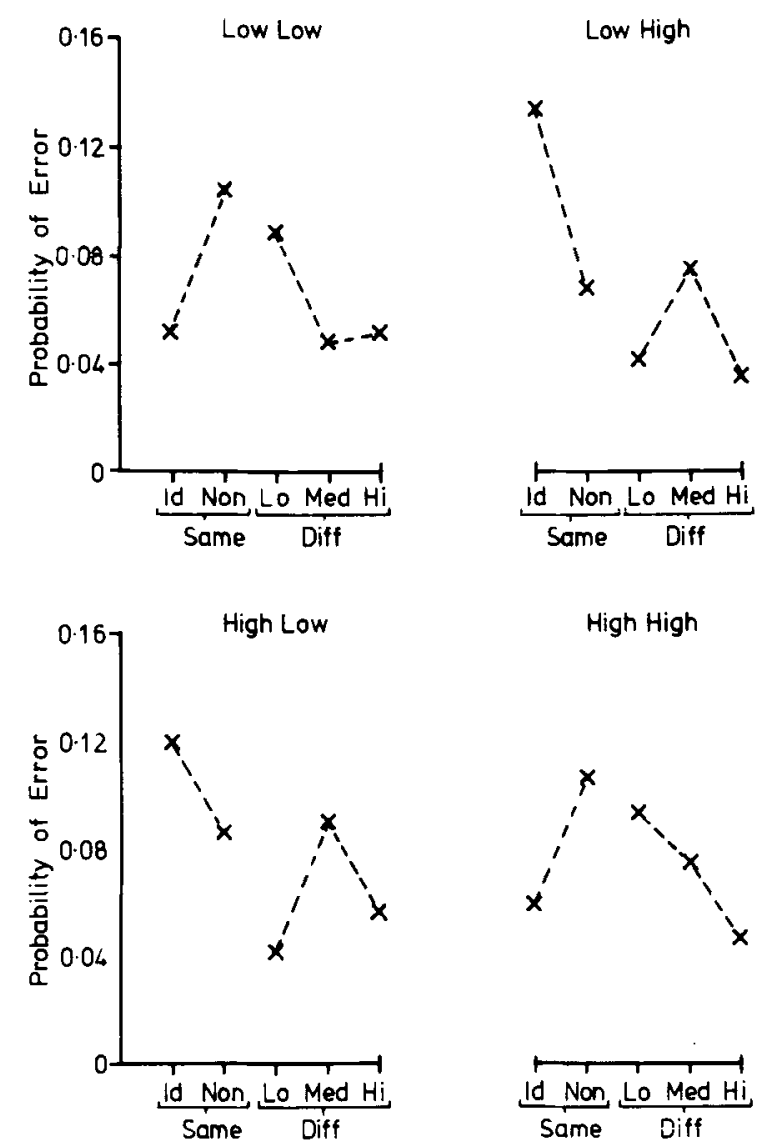

Figure 11. Error proportions for the matching conditions in the constant vowel set of Experiment 3. The label of each quadrant refers to the pitch of the first and second stimulus, respectively. $\mathrm{Hi}$, Med, and Lo refer to the amount of acoustic difference, as outlined in the text.

An alternative explanation of these results is that this may be determined by the process of comparison rather than by the properties of the memory. On this argument, while phonemes or subphonemic features constitute the auditory memory, syllables might be selected for comparison because these are presented within the structure of the syllable. This argument is reminiscent of McNeill and Lindig's (1973) demonstration that faster RTs to a list composed of syllable targets when a subject is monitoring for a syllable rather than for a phoneme does not provide support for the syllable as the unit of perception, but, rather, it reflects the difference between a match or mismatch between the targets and the search list at the linguistic level. Attributing the present results to the way the items are compared rather than to the way they are held in memory is less compelling. The formants of the vowel would not prevent matching at the auditory level because the vowel formant frequencies are the same for a given stimulus set. The different pitches on which the sounds are presented does not totally prevent matching at the auditory level for the constant vowel set. The parts of the syllable could in principle be compared without being prevented by a difference 
in pitch or in the formants of the vowel. However, in the varying vowel set (in which the vowel of the syllable changes when the sounds are presented on different pitches), the matching advantage is altogether abolished. The results do not appear to be due to the way the phonemes are packaged in the syllable; they appear to be due to the properties of the memory. Note that the memory need not be a linguistic categorization of the syllable but may be a characteristic of the spectral properties of the stimuli.

The cues to consonant phonemes are known to vary over syllables, and there is an obvious advantage in having all the cues to phonemes available from auditory memory for processing phonemes. However, it is not so widely known what information in a syllable gives extra information about the size of a speaker's vocal tract. The formant transitions are related to the rate at which the vocal cavities enlarge, and this information could be used to determine what phoneme was intended by that speaker.

Turning to the question of how pitch is transmitted for use by processing mechanisms beyond the auditory level, note two requirements of the process: First, from the findings of Experiment 1, matching at the auditory level of identical pairs that differ in pitch must be possible when the pitch range is small. Second, from the findings of Experiments 2 and 3, there must be the capacity to prevent subjects' doing this when the pitch range is larger for both stimulus sets. If the Morais and Darwin (1974) hypothesis that pitch and formants are represented separately at the auditory level is entertained, the findings of Experiment 1 are readily explicable. This can be extended to account for the findings of Experiments 2 and 3 by assuming that there is a feedback mechanism from the phonemic level which signals that decisions about formant frequencies which differ in pitch may lead to phoneme errors if auditory information (only the formants used for matching) is employed. Since this effect is vowel dependent, both the pitch range and the formant transitions influence decisions on auditory information. This dependence of phonemic decisions on both the formants and the pitch may account for why pitch is represented separately from the formants at the auditory level. The triggering of feedback at a given pitch could then be weighted for the formants separately from the pitch. A given pitch could prevent use of auditory information for some target frequencies and not for others.

The final question to be addressed is whether there is a separate normalization mechanism invoked when speech sounds are being dealt with from two or more simultaneous speakers. The change in vowel identity with pitch for the varying vowel set may be taken as evidence that different speakers produced those formant frequencies. It would be expected that if recalibration for different speakers gives an RT difference, then in Experiments 2 and 3, in which the speech has been identified as coming from different speakers, there would be a main effect of RT on vowel condition or an interaction with pitch of the pair. These are not observed, and it appears that recalibration for different speakers requires no extra time. Shankweiler, Strange, and Verbrugge (1977) proposed two hypotheses concerning this question. First, calibration of the perceptual mechanism for differences in the size of the speakers' vocal tracts could depend on prior exposure to speech from each speaker. Second, there could be cues in the consonant of the syllable that belong to the vowel just as much as to the consonant. Shankweiler et al. reasoned that if the former hypothesis were the case, there would be less difference in the identification of vowels in or out of consonant context if the listener were given speech from only one speaker than if there were more than one speaker. No difference was found between the pattern of results with one speaker and that with more than one speaker, and Shankweiler et al. consequently argued that no separate normalization exists. This explanation does not rule out the possibility that the vocal tract characteristics of a speaker who produced a syllable are extracted from the syllable. The mechanism automatically extracts information over a syllable-length segment to alter the calibration for another speaker. Thus the cues that the consonant shares with the vowel may be those that specify the articulatory geometry of the speaker.

In conclusion, the first experiment confirms the Morais and Darwin (1974) hypothesis that stimuli that differ moderately in pitch can be matched at the auditory level. The second and third experiments show that this is not true when a larger pitch range is used, particularly when the vowel of the syllable changes when pairs are on different pitches. The results are used to argue that syllables are held in auditory memory, and having this syllable context available gives additional information to categorize phonemes from different speakers. A means by which pitch information could be used selectively for phoneme processing is presented. Finally, no evidence was found in the present experiments for a separate normalization mechanism.

\section{REFERENCE NOTES}

1. Eimas, P. D., \& Miller, J. Auditory memory and the processing of speech (Brown University Progress Report No. 3). Providence, R.I: W. S. Hunter Laboratory of Psychology, 1975.

2. Repp, B. H. "Posner's paradigm" and categorical perception: A negative study (SR45/46). New Haven, Conn: Haskin's Laboratories, 1976.

\section{REFERENCES}

Darwin, C. J., Howell, P., \& Brady, S. A. Laterality and 
localization: A "right ear advantage" for speech heard on the left. In J. Requin (Ed.), Attention and performance VII. Hillsdale, N.J: Erlbaum, 1978.

Egeth, H. E. Parallel versus serial processes in multidimensional stimulus discrimination. Perception \& Psychophysics, 1966, 1, 245-252.

Fant, G., Carlson, R., \& Granstrom, B. The [e] - [ $\phi$ ] ambiguity. In G. Fant (Ed.), Speech communication seminar. Stockholm: Almqvist \& Wichsler, 1975.

HowELL, P. Syllabic and phonemic representations for short-term memory of speech stimuli. Perception \& Psychophysics, 1978, 24, 496-500.

Howell, P., \& Darwin, C. J. Some properties of auditory memory for rapid formant transitions. Memory \& Cognition, 1977, 5, 700-708.

Liberman, A. M., Cooper, F. S., Shankweiler, D. P., \& Studdert-Kennedy, M. Perception of the speech code. Psychological Review, 1967, 74, 431-461.

MCNeILL, D., \& Lindig, K. The perceptual reality of phonemes, syllables, words, and sentences. Journal of Verbal Learning and Verbal Behavior, 1973, 12, 419-430.

Mille R, R. L. Auditory tests with synthetic vowels. Journal of the Acoustical Society of America, 1953, 25, 114-121.

Morals, J. Monaural ear differences for same-different reaction times to speech with prior knowledge of ear stimulated. Perceptual and Motor Skills, 1975, 41, 829-830.

Morais, J., \& Darwin, C. J. Ear differences for same-different reaction times to monaurally presented speech. Brain and Language, 1974, 1, 383-390.

Pisoni, D. B., \& TASH, J. Reaction times to comparisons within and across phonetic categories. Perception \& Psychophysics, $1974,15,285-290$.

Posner, M. I., Boles, S. J., Eichelman, W. H., \& Taylor, R. L. Retention of visual and name codes of single letters. Journal of Experimental Psychology Monographs, 1969, 79.

Shankweiler, D., Strange, W., \& Verbrugge, R. Speech and the problem of perceptual constancy. In R. E. Shaw \& J. Bransford (Eds.), Perceiving, acting and knowing. Hillsdale, N.J: Erlbaum, 1977.

Studdert-Kennedy, M. The perception of speech. In T. A. Sebeok (Ed.), Current trends in linguistics. The Hague: Mouton, 1972.

WENDAHL, R. W. Fundamental frequency and absolute vowel identification. Journal of the Acoustical Society of America, 1959, 31, 109.110.

(Received for publication April 2, 1979; revision accepted November 26, 1979.) 